\title{
THREE-DIMENSIONAL EFFECTS IN MODELING OF DUAL-MODE SCRAMJETS
}

\author{
C.G. Rodriguez* \\ National Research Council, Hampton, VA 23681
}

\author{
J.A. White ${ }^{\dagger}$ \\ NASA Langley Research Center, Hampton, VA 23681
}

D.W. Riggins ${ }^{\S}$

University of Tennessee at Knoxville, TN 37996

\begin{abstract}
$\underline{\text { Abstract }}$
A numerical investigation of an experimental dual-mode scramjet configuration is performed. Both experimental and numerical results indicate significant upstream interaction for this case. Several computational cases are examined: these include the use of jet-to-jet symmetry and entire halfduct modeling. Grid convergence, turbulence modeling, and wall temperature effects are studied in terms of wall pressure predictions and flow-field characteristics. Wall pressure comparisons between CFD and experiment show fair agreement for the jet-to-jet case. However, further computations of the entire half-duct show the development of a large sidewall separation zone extending much further upstream than the separation zone at the duct centerline. This sidewall separation is the dominant feature in the CFD-generated flowfield but is not evident in the experimental data, resulting in a unfavorable comparison between CFD and experimental data. Current work aimed at resolving this issue and at further understanding asymmetric flow-structures in dual-mode flow-fields is discussed.
\end{abstract}

* Research Associate, Member AIAA

$\dagger$ Aerospace Engineer, Hypersonic Airbreathing Propulsion Branch.

$\S$ Associate Professor, Senior Member AIAA.

Copyright $\odot 2000$ American Institute of Aeronautics and Astronautics, Inc. All rights reserved.

\section{$\underline{\text { Nomenclature }}$}

$\begin{array}{ll}\mathrm{h}_{\mathrm{isol}} & \text { isolator height }[\mathrm{m}] \\ \mathrm{k} & \text { turbulence kinetic energy }\left[\mathrm{m}^{2} / \mathrm{s}^{2}\right] \\ \mathrm{M} & \text { Mach number } \\ \mathrm{p} & \text { pressure }[\mathrm{Pa}] \\ \mathrm{T} & \text { temperature }\left[{ }^{\circ} \mathrm{K}\right] \\ \mathrm{x} & \text { axial (streamwise) coordinate }[\mathrm{m}] \\ \mathrm{y} & \text { vertical coordinate }[\mathrm{m}] \\ \mathrm{y}_{\mathrm{i}} & \text { mass-fraction of specie } \mathrm{i} \\ \mathrm{y}^{+} & \text {dimensionless vertical turbulence coordinate } \\ \mathrm{z} & \text { lateral coordinate }[\mathrm{m}] \\ \phi & \text { equivalence ratio } \\ \mu & \text { viscosity }\left[\mathrm{N} \text { s/m }{ }^{2}\right] \\ \eta_{\mathrm{c}} & \text { combustion efficiency } \\ \eta_{\mathrm{m}} & \text { mixing efficiency } \\ \rho & \text { mass density }\left[\mathrm{kg} / \mathrm{m}^{3}\right] \\ \omega & \text { specific dissipation rate }[1 / \mathrm{s}] \\ & \\ \mathrm{Subscripts}: & \\ 0 & \text { total conditions } \\ \mathrm{j} & \text { injectant (jet) conditions } \\ \mathrm{s} & \text { static conditions } \\ \infty & \text { free-stream (inlet) conditions } \\ \end{array}$

\section{Introduction}

The flow-field in a dual-mode scramjet is transistional between the low-speed flight regime (vehicle flight Mach below approximately 5) and the high-speed flight regime (vehicle flight Mach number above approximately 8). For this reason, the operating range for a scramjet between flight Mach 5 and Mach 8 is called the mid-speed range or dual-mode regime. In this range engine performance is characterized by complex transistional fluid dynamics, intermediate between the two limits of a completely subsonic combustor flow-field and a completely supersonic combustor flow-field. In the case of the sub- 
sonic (low-speed) limit, engine throttling determines upstream component (inlet) performance and mass capture. This occurs most generally through the mechanism of shock waves located inside the inlet for supercritical operation and external to the inlet for subcritical operation. The placement of these shocks is driven largely by the ratio of the totalenthalpy flux entering the engine to the heat release in the subsonic combustor. Conversely, for a completely supersonic combustor flow-field (high-speed case), inlet performance and mass capture are independent of engine throttling in general, i.e. the flow-field of components upstream of the combustor are not affected by the heat release in the combustor. However, as flight Mach number is lowered for a scramjet, the total enthalpy flux entering the combustor is lowered such that the ratio of heat released to entering enthalpy climbs rapidly. Eventually, flow-field interactions upstream of the combustor begin to take place. A scramjet designed for high-speed operation will unstart when operated in the mid-speed region, i.e. a shock will establish itself at a location external to the engine with corresponding large changes in performance, unless suitable design changes are made.

The most common design features which allow for started dual-mode scramjet operation include 1) small backward facing steps located forward in the combustor which tend to contain the combustion-induced pressure rise aft of the steps, 2) area expansion in the combustor in order to maintain flow expansion and delay the formation of thermal choke, 3) the addition of a constant-area duct upstream of the combustor in order to allow some limited upstream interaction without necessarily affecting the inlet (this component is termed the isolator due to its tendency to isolate the inlet from the combustor) and 4) staged injection in which fueling is performed at various axial locations depending on the flight regime in order to control heat release schedule and location. All of these features are generally regarded as necessary for successful dual-mode operation.

The mechanism of upstream pressure rise in a dualmode system with isolator occurs through the formation and evolution of an upstream oblique shock-train system (see figure 1). This shock system forms in the isolator and is associated with the development of upstream recirculation zones on isolator walls; as the engine is throttled (or equivalently as downstream heat release/pressurization increases), these recirculation zones with their associated shock structures move forward, hence increasing the extent and magnitude of the upstream pressure rise. It is critical for engine design and for successful engine operation to properly understand the physics of the upstream interaction in a dual mode scramjet engine. However, the flow-field in the isolator- combustor for dual-mode operation is complex and highly variable; the core flow can be entirely subsonic or remain supersonic. Broad features of the flow are driven by relatively small effects such as incoming boundary-layer characteristics. Due to the large recirculation bubbles present in the flow, three-dimensional characteristics can be significant and coupling of these recirculation regions with walls and corner flows can cause significant lateral distortion.

The dual-mode flow-field therefore represents a highly-challenging problem for computational fluid dynamics. This is due to 1) the strongly elliptical nature of the flowfield associated with the extensive upstream interaction, 2) the related importance of high-level turbulence modeling necessary to predict the extent and shape of that interaction, 3) downstream mixing and combustion at low(er) Mach number, 4) the simultaneous presence of large bulk subsonic and supersonic regions embedded within the combustor/isolator, and 5) possible unsteadiness and resulting asymmetric flow features in geometrically symmetric configurations.

The specific purposes of this paper are to investigate numerically a) grid refinement issues, b) wall temperature effects, and c) turbulence model effects for a selected dualmode configuration with experimental data. In addition, sidewall effects on the same flow-field are studied. Differences between computational modeling and experimental data are discussed and analyzed.

Previous work in the area of dual-mode analysis and predictive capability includes the early work of Billig ${ }^{1}$, Billig et $\mathrm{al}^{2}$, and Waltrup and Billig3 ${ }^{3}, 4$. They developed correlations based on one-dimensional and two-dimensional flow analysis which included such drivers as incoming boundary-layer thickness and maximum combustor pressure rise. These correlations perform well on simple model geometries (axi-symmetric or two-dimensional) but are not generally suitable for more complex three-dimensional problems except for providing directions and trends. Experimental studies at the National Aerospace Laboratory (NAL) in Japan have been performed by Komuro et $\mathrm{al}^{5,6}$ and further reported on by Chinzei et $\mathrm{al}^{7}$. Recent computational studies of the same configuration are described in papers by Mizobuchi et $\mathrm{al}^{8}$ and Matsuo et $\mathrm{al}^{9}$. This experiment, featuring a direct-connect scramjet combustor-isolator rig, has been chosen for analysis in the present paper.

The second section of this paper provides a brief description of the experimental configuration of the NAL scramjet. The third section of the paper is a review of the computational method (code) used in the present study. The fourth section presents computational methodology and results of the numerical study with comparisons to experimental data and subsequent analysis of the discrep- 
ancies between experimental data and the numerically-predicted flow-fields. Finally, the last section provides a summary of the investigation, ongoing work, and recommendations regarding the numerical simulation of dual-mode flow-fields.

\section{Description of the Experiment}

This section briefly describes the experiment examined in this investigation; greater detail can be found in the previously-mentioned references ${ }^{5,6,7}$.

The scramjet is essentially a rectangular-section, constant-width duct; the cross-section aspect-ratio is approximately 4.60. On both upper and lower walls there is a backward-facing step and, further downstream, a $1.7^{\circ}$ wall expansion. These two features divide the scramjet in three sections: isolator, combustor and expansion. In the following description the isolator height $\mathrm{h}_{\text {isol }}(=0.032 \mathrm{~m})$ will be used as reference length; actual dimensions can be obtained from figure 2 .

The steps have a height of $0.10 \mathrm{~h}_{\text {isol }}$. Injection takes place $0.40 \mathrm{~h}_{\text {isol }}$ downstream of the steps. There are five injectors on the bottom wall, and four on the top; the top injectors are interdigitated with respect of the bottom ones. The two side orifices on the bottom wall are half the area of the others; this arrangement is intended to provide the same fuel flowrate on both walls.

A vitiated air-heater provides the inflow to the scramjet through a Mach 2.5 (nominal) facility nozzle; this is intended to represent a flight condition of Mach 7.5. As will be explained subsequently, this nozzle was simulated numerically and the exit conditions used as inflow for the present calculations.

Hydrogen is injected through the orifices at sonic conditions, with a total temperature of $280{ }^{\circ} \mathrm{K}$. In the experiment the fuel flowrate was varied for equivalence ratios $\phi$ that ranged from 0 to 1.0; actual injection conditions will be described later in this paper.

\section{Description of the VULCAN Code}

VULCAN $^{10}$ (a Viscous Upwind aLgorithm for Complex flow ANalysis) is a cell-centered finite-volume, structured grid, multi-block code which solves the equations governing inviscid and viscous flow of a calorically perfect gas or of an arbitrary mixture of thermally perfect gases undergoing nonequilibrium chemical reactions. VULCAN allows the flow domain to be decomposed into regions in which the most suitable algorithm (elliptic or marching) can be utilized.

The inviscid fluxes are computed using a 2nd- or 3rdorder accurate MUSCL scheme ${ }^{11}$ with either the approximate Riemann solver of Roe ${ }^{12}$ or the low-dissipation fluxsplitting scheme of Edwards ${ }^{13}$. The viscous fluxes can be evaluated either with or without cross derivative contributions using second order central differences.

The equations governing spatially-elliptic flows are solved by marching their unsteady form in time while the equations governing spatially-hyperbolic (e.g. the Euler equations in supersonic flow) and spatially-parabolic flows (e.g. the parabolized Navier-Stokes equations) are solved by marching their unsteady form in space while locally iterating in pseudo-time to a steady state solution. The four schemes available for marching in physical or pseudo-time are: a multi-stage Runge-Kutta scheme, a diagonalized approximate factorization scheme, a block approximate factorization scheme, and a diagonally dominant alternating direction implicit scheme.

Several two-equation turbulence models are implemented in VULCAN, in such a manner as to allow flexibility when modeling the Reynolds stresses. If one of the eddy-viscosity based two equation turbulence models is selected, the Reynolds stresses are modeled using the Boussinesq approximation. However, if an explicit algebraic Reynolds stress two-equation turbulence-model is selected, then the Reynolds stresses are modeled with a non-linear constitutive relation ${ }^{15,16,17}$.

The two-equation turbulence models in VULCAN can be categorized as k- $\varepsilon$ based models, Wilcox's k- $\omega$ based models and Menter's k- $\omega$ models. The k- $\varepsilon$ based models can be solved with either the Boussinesq model or an explicit algebraic Reynolds stress model ${ }^{14}{ }^{16}$. The Wilcox $\mathrm{k}-\omega$ based models are available in three forms ${ }^{18}$ : a high Reynolds number model, a low Reynolds number model, and an explicit algebraic Reynolds stress model. The k- $\omega$ models of Menter ${ }^{14}$ are available in two forms: the baseline model (BSL) and the Shear Stress Transport (SST) model.

A correction to each model for compressibility effects is also implemented using the compressible dissipation model of Sarkar et al. for k- $\varepsilon$ based models ${ }^{18}$, and the compressibility model suggested by Wilcox ${ }^{18}$ (which incorporates Sarkar's model as well as Zeman's lagging function) for all of the k- $\omega$ based models (including Menter's).

The near-wall behavior of the k- $\varepsilon$ two equation models is controlled through the introduction of the low Rey- 
nolds number modifications of $\mathrm{Abid}^{15}$. The near-wall behavior of the k- $\omega$ two equation models is treated either by integrating the high-Reynolds number form of the equations to the wall (without any special wall treatment), or by integrating the low Reynolds form of the Wilcox k- $\omega$ two equation model to the wall.

If integration to the wall is not feasible for a given problem, the wall matching function approach of Wilcox ${ }^{18}$ can be used. This approach models the wall shear stress and heat transfer by enforcing a compressible law of the wall which includes additional terms that model streamwise pressure gradients. Wilcox showed that inclusion of the pressure gradient terms in the wall matching function dramatically improved predictions of shear stress.

\section{Results}

For the purposes of the present investigation, two computational domains will be considered: a jet-to-jet domain (described below), and a domain comprising half the crosssection shown in figure 2. Following are the characteristics common to both simulations.

The domain extends from the inlet of the isolator to $12.50 \mathrm{~h}_{\text {isol }}$ downstream of the steps. This length is shorter than that of the experimental duct, but is sufficiently long to provide supersonic conditions at the exit.

Inlet conditions were obtained from the numerical simulation of the facility nozzle. For convenience, the nominal inlet conditions of the vitiated flow are provided in table 1. For the jet-to-jet domain, the inlet centerplane-profiles were propagated along the width of the domain. Exit conditions were set as supersonic extrapolation.

\begin{tabular}{|c|c|}
\hline $\mathrm{T}_{0}\left[{ }^{\circ} \mathrm{K}\right]$ & 2000 \\
\hline $\mathrm{p}_{0}[\mathrm{MPa}]$ & 1.0 \\
\hline$\rho_{0}\left[\mathrm{Kg} / \mathrm{m}^{3}\right]$ & 1.58 \\
\hline $\mathrm{y}_{\mathrm{N} 2}$ & 0.5832 \\
\hline $\mathrm{y}_{\mathrm{O} 2}$ & 0.24335 \\
\hline $\mathrm{y}_{\mathrm{H} 2 \mathrm{O}}$ & 0.17315 \\
\hline
\end{tabular}

Table 1: Nominal inlet conditions.

Unless otherwise noted, all solid walls are modeled as no-slip and isothermal, with constant wall-temperature set at $500^{\circ} \mathrm{K}$ based on information provided to the authors by the NAL researchers.
The property profiles at the injectors were assumed to be uniform. Hydrogen is injected at sonic conditions, with conditions given in table 2 . No fuel flowrates are quoted in the references; rather, they are given in terms of the equivalence ratio $\phi$ (with a discharge coefficient of 0.85 assumed for all injectors). Therefore, for the present calculations the fuel flowrates were determined as functions of the numerical inlet flowrates. The flowrates, in turn, determine the injectant density as a function of the global $\phi$. The corresponding values will differ for the two computational domains and are given below.

\begin{tabular}{|l|c|}
\hline $\mathrm{M}_{\mathrm{j}}$ & 1.0 \\
\hline $\mathrm{T}_{0 \mathrm{j}}\left[{ }^{\circ} \mathrm{K}\right]$ & 280 \\
\hline $\mathrm{T}_{\mathrm{s} \mathrm{j}}\left[{ }^{\circ} \mathrm{K}\right]$ & 233 \\
\hline $\mathrm{u}_{\mathrm{j}}[\mathrm{m} / \mathrm{s}]$ & 1161 \\
\hline$\rho_{\mathrm{j}}\left[\mathrm{kg} / \mathrm{m}^{3}\right]$ & see text \\
\hline $\mathrm{k}_{\mathrm{j}}\left[\mathrm{m}^{2} / \mathrm{s}^{2}\right]$ & .01 \\
\hline$\mu_{\text {Turb }} / \mu_{\text {Lam }}$ & 1.0 \\
\hline
\end{tabular}

Table 2: Injectant conditions.

Unless otherwise noted, all cases were run with the following code methodology (which defines a baseline condition). The inviscid fluxes were calculated with the low-dissipation flux-split scheme, with MUSCL thirdorder interpolation and Van-Leer limiter. Menter's k- $\omega$ baseline (BSL) turbulence model was used, with Wilcox' wall-matching and compressibility-correction functions. The flow was modeled as a chemically-reacting mixture of thermally-perfect gases. Both turbulent Prandtl and Schmidt numbers were set at 0.50 ; these values are consistent with shear-dominated flows. Finite-rate chemistry was simulated with a 7-specie, 7-equation model. Time integration was performed using the implicit, diagonal approximate-factorization scheme, with local time-stepping. Most of the cases were run on a Cray c90 supercomputer, using a single processor. CPU times were approximately $120 \mu \mathrm{s} /$ iteration/point. CFL numbers ranged from 0.1 to 5.0. Convergence was considered achieved when no changes were observed in the wall-pressure traces, which usually required between 15,000-20,000 iterations. 


\subsection{Jet-to-Jet Domain}

For this case the domain was restricted from lower-jet centerline to upper-jet centerline. Furthermore, the upperhalf of the domain was replaced with an anti-symmetric boundary at the horizontal centerplane. The numerical inlet flowrate was found to be $7.226 \times 10^{-2} \mathrm{~kg} / \mathrm{s}$. All cases were run with $\phi=1$, which results in $\rho_{\mathrm{j}}=0.286 \mathrm{~kg} / \mathrm{m}^{3}$.

Three grids were used for the jet-to-jet calculations. Their dimensions are given in table 3 in the form axial $\times$ vertical $\times$ lateral. Unless otherwise noted, all results to be shown in this section correspond to the medium grid. For the present conditions, this grid has a $\mathrm{y}^{+}$at the wall of less than 100 , which is adequate for the use of wall-matching functions.

\begin{tabular}{|l|c|c|}
\hline \multicolumn{1}{|c|}{ Grids } & Isolator & $\begin{array}{c}\text { Combustor\& } \\
\text { expansion }\end{array}$ \\
\hline \hline Coarse & $101 \times 13 \times 13$ & $103 \times 19 \times 13$ \\
\hline Medium & $149 \times 21 \times 31$ & $185 \times 33 \times 31$ \\
\hline Fine & $201 \times 29 \times 41$ & $277 \times 45 \times 41$ \\
\hline
\end{tabular}

Table 3: Computational grids for jet-jet domain.

Figure 3 shows the centerplane Mach contours for the baseline condition (for better resolution, the vertical scale is twice the horizontal). For this high- $\phi$ condition the degree of upstream interaction is substantial, reaching about $4 \mathrm{~h}_{\text {isol }}$ upstream of the steps. The recirculation bubbles and the shock-train, typical of dual-mode combustion, are clearly seen. The flow chokes in the vicinity of the steps and reaccelerates to supersonic at the beginning of the expansion.

Wall pressures at the bottom on the centerplane are compared in figure 4 with the data of reference 5 . The extent of the upstream interaction is close to the experimental value, but the peak pressure is overpredicted by about $15 \%$.

The mixing and combustion efficiencies are shown in figure 5 with the corresponding experimental values. For the jet-to-jet domain, the efficiencies are overpredicted with respect to the data. Also, the difference between the two efficiencies is greater than indicated in the experiment.

In order to study grid refinement, a comparison between the grids described in table 3 was made. The results are shown in figure 6 in terms of the wall pressures. Grid convergence appears to have been achieved; the medium grid is adequate for the present studies, and will provide the baseline reference. On the other hand the coarse grid, while quantitatively inadequate, gives acceptable qualitative results. This suggest that coarse grids can be used as a first approximation in order to study the qualitative behavior of a given flowfield, before committing to a more accurate (and costly) calculation.

Since there is some uncertainty regarding the actual wall temperatures, the sensitivity to wall conditions was explored. Figure 7 compares the wall pressures for the isothermal condition used with an adiabatic one. The extent of the upstream interaction appears to be the same, while the adiabatic case shows somewhat lower peak-pressures. It is possible that the thinner boundary-layer in the isothermal case causes a greater turning-angle at the leading-edge of the bubble, resulting in a stronger shock. However, both conditions give similar results. This would suggest that some uncertainty can be tolerated in the definition of the conditions at the walls, at least for this problem.

Figure 8 shows the impact of the different versions of the k- $\omega$ turbulence model. They all predict the same qualitative behavior. However, Wilcox' 1998 model reduces the pressure overprediction by half to $7 \%$. The algebraic-stress model (EASM) somewhat improves the prediction of the extent of upstream interaction (note, however, the latter model is still in development within VULCAN).

While working on the half-width calculations (to be shown in the following subsection) a curious anomaly was encountered, which is presented in figures 9 and 10 (for a coarse grid with baseline conditions). When the antisymmetric boundary is removed and the entire height solved, the resulting flowfield is not antisymmetric (even though the domain, grid and boundary-conditions are). Figure 9 shows that the lower recirculation bubble dominates the upper one; other calculations, with small changes in the set-up, may show the opposite. The overall upstream interaction is bigger, and the peak-pressure smaller, than predicted by the half-height calculation (figure 10); the impact of the shock-train on the upper-wall is apparent. This phenomena may be explained as the 'buckling' of the 'inviscid' core flow between the bubbles. It appears to occur when the length of the upstream interaction exceeds 1-2 times the height of the domain. Further work is under way to understand this behavior.

\subsection{Half-width Domain}

Fuel contour-plots from reference 7 show a considerable amount of three dimensionality. Specifically, excess 
fuel appears to concentrate in the middle of the flow, suggesting that the inflow is being pushed towards the sidewalls. This may result in lower pressures at the centerplane, as well as lower efficiencies. In any case, this effect puts into question the lateral-symmetry assumption implicit in the jet-to-jet calculations.Therefore, it was decided to model the entire half of the domain to see if this behavior could be reproduced. Obviously, this required the use of a symmetry boundary to represent the other half. Referring to what was said at the end of the previous sub-section, numerical experimentation showed that the flowfield is actually symmetric with respect to the centerplane, which therefore is taken as a true symmetry plane.

For the half-width domain the numerical inlet flowrate was found to be $0.66 \mathrm{~kg} / \mathrm{s}$. The equivalence ratio was reduced to $\phi=0.80$, because of concerns of the interaction going into the nozzle (as will be discussed shortly). The resulting density for all injectors was $\rho_{\mathrm{j}}=0.266 \mathrm{~kg} / \mathrm{m}^{3}$.

The grid used for the present calculations was $101 \times 25$ $\times 63$ for the isolator, and $103 \times 37 \times 63$ for the combustor and expansion. This grid is equivalent to the coarse grid used in the jet-to-jet calculations. Therefore, it is expected that it will provide adequate qualitative information in anticipation of a grid-refined calculation. The rest of the code methodology is the same as in the jet-to-jet baseline condition.

The Mach contours for this case are shown in figure 11; in this view, the symmetry centerplane is at the back while the sidewall is nearest to the viewer. CFD predicts a massive upstream interaction on the sidewall, and a much smaller (and discontinuous) one at the centerplane; this result is clearly at odds to what the jet-to-jet calculations (and the data) suggest. Note that the sidewall bubble is very close to the inlet. Apparently, this bubble pushes the inflow towards the centerplane, and as a result it "sweeps" the downstream portion of the center bubble; this may account for the shorter length of the latter. The discontinuous nature of the centerplane upstream-interaction is reflected in the wall-pressure trace (figure 12). Clearly the wall pressure is far below the data, which is a result of the shorter centerplane bubble.

The fuel contour-plots and efficiencies can be seen in figures 13 and 14, respectively. As could be expected from the previous discussion, the fuel tends to be pushed towards the sidewall in direct contrast to the experimental results. This relatively large amount of fuel entrained near the sides probably accounts for the lower numerical efficiencies (at least for the combustor section). It should be noted that the efficiencies shown correspond to the $\phi=1$ condition.

\section{Conclusions and Future Work}

A dual-mode scramjet combustor configuration which has experimental data indicating substantial upstream interaction has been modeled numerically. Results show the development of a significant oblique shock/expansion train upstream of the combustor. Several computational studies are discussed in this investigation; a sub-domain using jet-to-jet symmetry was first modeled and effects of grid refinement, wall temperature, and turbulence models were examined. Predicted wall pressures for all these cases are in reasonable agreement with the experimental data with the best relative performance exhibited by both the Wilcox $1998 \mathrm{k}-\omega$ turbulence model and, in particular, the explicit algebraic-stress model (EASM). Grid convergence is conclusively demonstrated for this study. Cold wall and adiabatic wall treatment yield similar results in terms of wall pressure prediction and extent of upstream interaction; small differences may be attributed to boundary layer effects and incident shock angles at the leading edge of the upstream separation bubbles. Overall, comparisons between CFD and experimental data indicate a slight overprediction in peak wall pressure and a slight underprediction of upstream interaction distance.

Qualitative fuel/air contour plots obtained experimentally indicate that there is a bulk movement of the air around the fuel-injector array (toward the side walls) resulting in a fuel-rich core along the duct centerline. This is a possible explanation for the higher wall pressures along the centerline for the CFD; the CFD, using jet-to-jet symmetry, cannot capture this bulk lateral movement of the air and hence fuel/air reaction occurs nearer to stoichiometric conditions at the centerline.

Due to these three-dimensionality concerns, a study was initiated in which the entire half-width of the duct was modeled. Wall pressures and upstream interaction are significally underpredicted for this case; in fact, the data comparison is considerably degraded from that achieved with jet-to-jet symmetry even though nominally the lateral movement of the air should be better captured. The reason for this discrepancy is that the CFD predicts the development of a large forward recirculation zone on the isolator sidewall. This sidewall separation directs air toward the centerline of the duct. Note that there is no indication of this effect in the experimental data. In fact, just the opposite is seen to occur (there is a bulk movement of the incoming air toward the sidewall which indicates little or no significant additional separation on the isolator sidewall). Several possibilities have been and are being investigated to explain this discrepancy. Primary focus at this time is on turbulence modeling associated with corner flows; important secondary-flow features are not predicted 
when using standard two-equation turbulence models. Relatively large vortices associated with the secondary flows in the corners near the sidewall should significantly reduce the extent of flow separation along the sidewall. However, recent computations using the EASM model (which offers better prediction capability for such flows) have not shown any significant impact on the side-wall separation phenomena observed in the CFD. This problem is currently under study.

In addition to the side-wall separation issue discussed above, related studies with regards to the use of symmetry and anti-symmetry boundary planes have indicated the development of asymmetric flow structure between top and bottom regions in geometrically symmetric two-dimensional test cases as well as in the three-dimensional problem discussed in this investigation. Such cases are characterized by stronger shocks on one wall than on the other (top to bottom) and occur primarily when upstream recirculation zones are sufficiently large. Note that such flows are in equilibrium, i.e. the effect is observed at steady-state. Several possibilities are being examined as candidates to explain this effect. Levy et $\mathrm{al}^{19}$ contend that some time-integration algorithms (particularly the diagonal approximate factorization used here) are not symmetry-preserving, i.e. small non-symmetric error can induce eventual development of asymmetric flow-fields in symmetrically-constrained (symmetric geometry and boundary conditions) problems; such behavior is seen in various flows such as slender bodies at high angles of attack and can mimic transient-disturbance induced asymmetry observed experimentally. However, in the present investigation, the experimental wall pressure data indicates no discernible asymmetry. There is, however, experimental evidence in similar cases ${ }^{20}$ indicating that upstream shock trains on backpressured geometries can, in fact, develop and stabilize with asymmetric features very similar to what has been observed in the CFD generated flow-fields in this investigation. Currently, studies are underway to clarify whether this effect is primarily a physical issue or a modeling issue.

\section{Acknowledgments}

This investigation was supported through the National Research Council by NASA Grant NASW-4907, with C. McClinton from NASA Langley as technical advisor.

\section{$\underline{\text { References }}$}

1 Billig, F.S. and Dugger, G.L., "The interaction of Shock Waves and Heat Addition in the Design of Supersonic Combustors", Proceedings of the $12^{\text {th }}$ Symposium on Combustion, Combustion Institute, Pittsburgh, PA, 1969, pp. 1125-1134.

2 Billig, F.S, Dugger, G.L., and Waltrup, P.J., "Inlet-Combustor Interface Problems in Scramjet Engines", Proceedings of the $1^{\text {st }}$ International Symposium on Airbreathing Engines, Marseilles, France, June 1972.

3 Waltrup, P.J. and Billig, F.S, "Prediction of Precombustion Wall Pressure Distribution in Scramjet Engines", Journal of Spacecraft and Rockets, Vol. 10, No. 9, 1973, pp. 620-622.

4 Waltrup, P.J. and Billig, F.S, "Structure of Shock Waves in Cylindrical Ducts", AIAA Journal, Vol. 11, No. 9, 1973, pp. 1404-1408.

5 Komuro, T., Kudo, K. Masuya, G., Chinzei, N., Murakami, A., and Tani, K., "Experiment on a Rectangular Cross Section Scramjet Combustor" (in Japanese), National Aerospace Lab, NAL TR-1068, Tokyo, Japan, 1990.

6 Murakami, A., Komuro, T., and Kudo, K., "Experiment on a Rectangular Cross Section Scramjet Combustor (II) - Effects of Fuel Injector Geometry", (in Japanese), National Aerospace Lab, NAL TR-1220, Tokyo, Japan, 1993.

7 Chinzei, N., Komuro, T., Kudou, K., Murakami, A., Tani, K., Masuya, G. and Wakamatsu, Y., "Effects of Injector Geometry on Scramjet Combustor Performance", AIAA Journal of Propulsion and Power, Vol. 9, No. 1, Jan.-Feb. 1993, pp. 146-152.

8 Mizobuchi, Y., Matsuo, Y. and Ogawa, S., "Numerical Estimation of Turbulence Temperature Fluctuation Effect on Hydrogen-Oxygen Reaction Process", AIAA Paper 97-0910, January 1997.

9 Matsuo, Y., Mizobuchi, Y. and Ogawa, S., "Parallel Numerical Simulation of Compressible Free Shear Layers in a Scramjet Engine", AIAA Paper 98-0963, January 1998.

10 White, J.A., Morrison, J.H., "A Pseudo-Temporal Multi-Grid Relaxation Scheme for Solving the Parabolized Navier-Stokes Equations", AIAA 99-3360, 1999.

11 van Leer, B., "Towards the Ultimate Conservative Difference Scheme V. A Second Order Sequel to Gudunov's Method", Journal of Computational Physics, Vol. 32, pp. 101-136, 1979.

12 Roe, P.L., "Discrete Models for the Numerical Analysis of Time-Dependent Multidimensional Gas Dynamics", Journal of Computational Physics, Vol. 63, pp. 458-476, 1986. 
13 Edwards, J.R., "A Low-Diffusion Flux-Splitting Scheme for Navier-Stokes Calculations", Computers and Fluids, Vol. 26, No. 6, pp. 635-659, June 1997.

14 Menter, F.R., "Improved Two-Equation k-w Turbulence Model for Aerodynamic Flows", NASA TM-103975, 1992.

15 Abid, R., Morrison, J.H., Gatski, T.B., and Speziale, C.G., "Prediction of Complex Aerodynamic Flows with Explicit Reynolds Stress Models", AIAA 96-0565, 1996.

16 Gatski, T.B. and Speziale, C.G., "On Explicit Algebraic Stress Models for Complex Turbulent Flows”, NASA-CR189725.

17 Abid, R., Rumsey, C.L., and Gatski, T.B., "Prediction of Nonequilibrium Turbulent Flows with Explicit Algebraic Turbulence Models", AIAA Journal, Vol. 33, No. 11, Nov. 1995.

18 Wilcox, D.C., Turbulence Modeling for CFD, 2nd Edition, DCW Industries, Inc., 1998.

${ }^{19}$ Levy, Y., Hesselink, L., and Degani, D., “Anomalous Asymmetries in Flows Generated by Algorithms that Fail to Conserve Symmetry", AIAA Journal, Vol. 33, No. 6, June 1995, pp. 999-1007.

${ }^{20}$ Carrol, B.F., and Dutton, J.C., "Characteristics of Multiple Shock Wave/Turbulent Boundary Layer Interactions in Rectangular Ducts", Journal of Propulsion and Power, Vol. 6, No. 2, March-April 1990, pp. 186-193. 


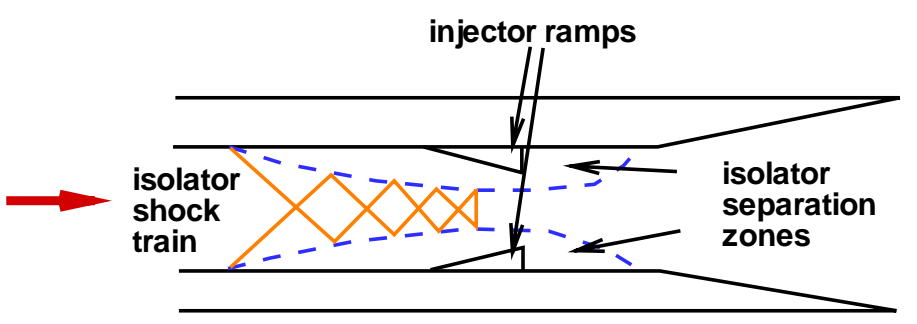

Figure 1: Schematics of the flowfield in a dual-mode combustor.

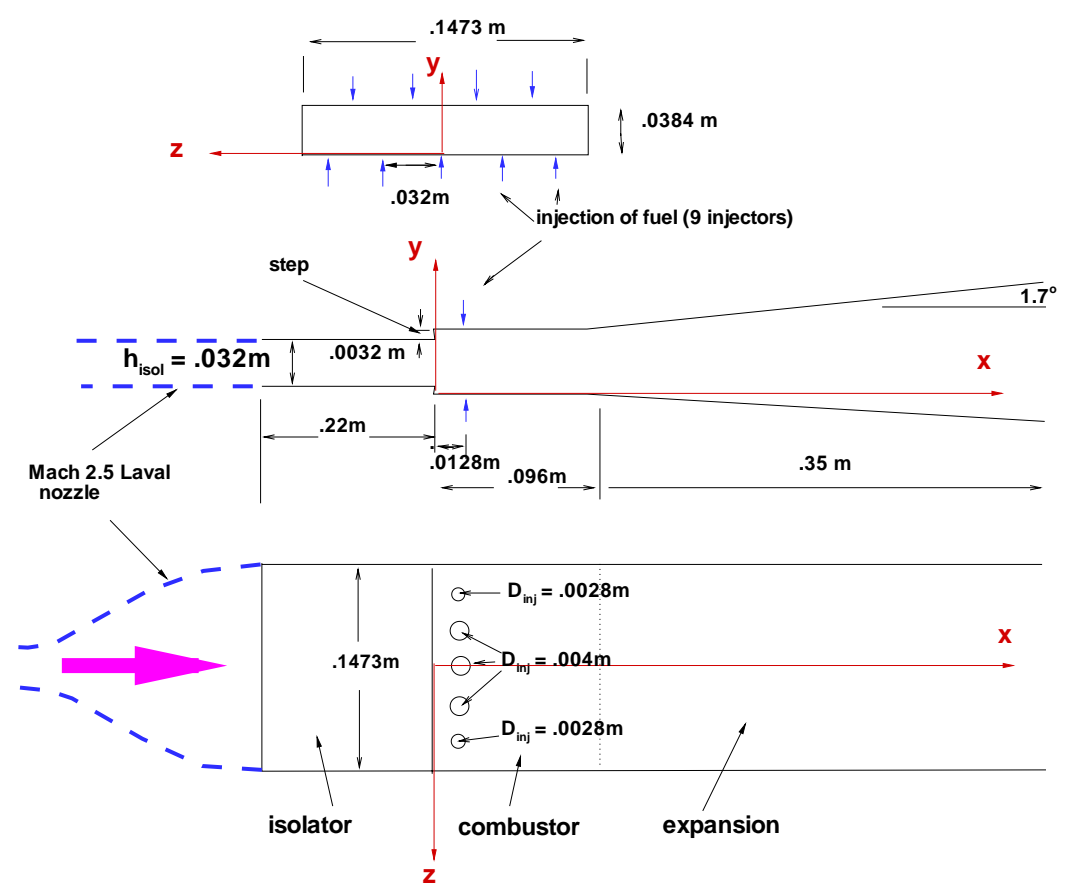

Figure 2: Outline of the NAL dual-mode scramjet experiment. 


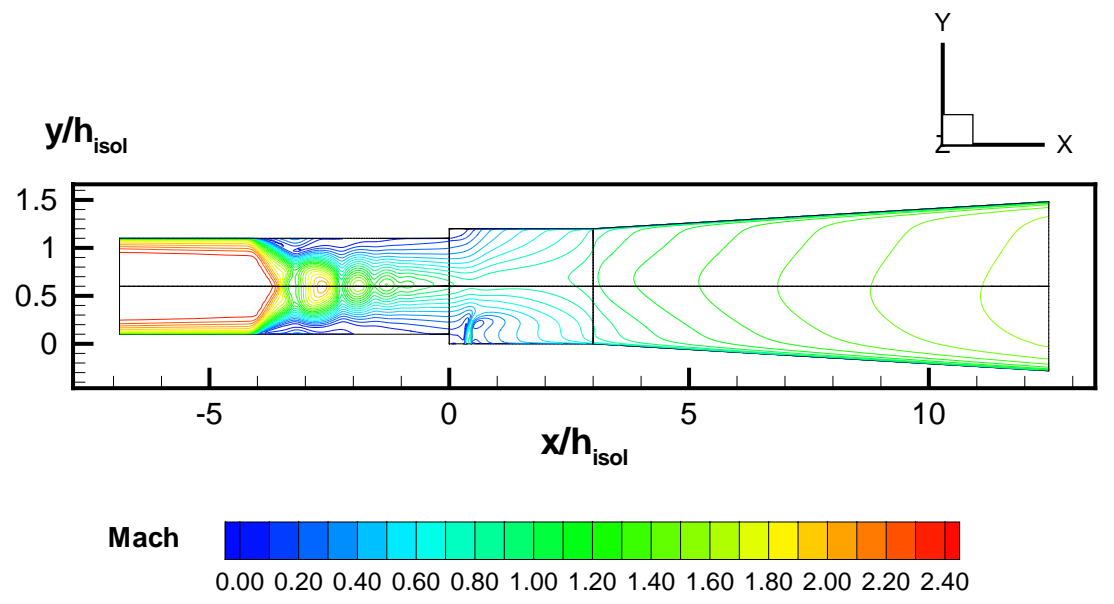

Figure 3: Jet-to-Jet - Mach contours (baseline condition).

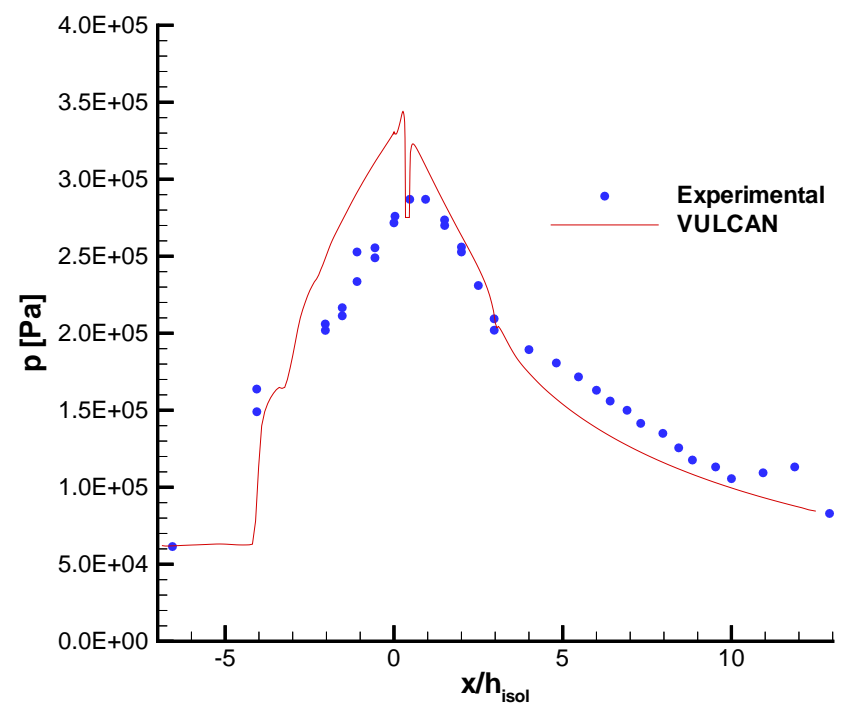

Figure 4: Jet-to-Jet - Wall pressures (baseline condition). 


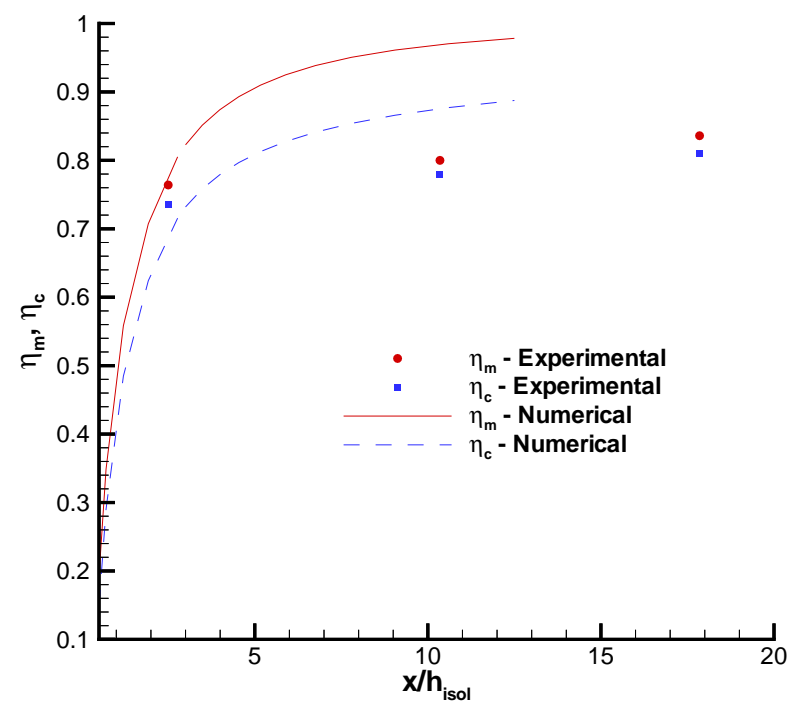

Figure 5: Jet-to-Jet - Mixing and reacting efficiencies (baseline condition).

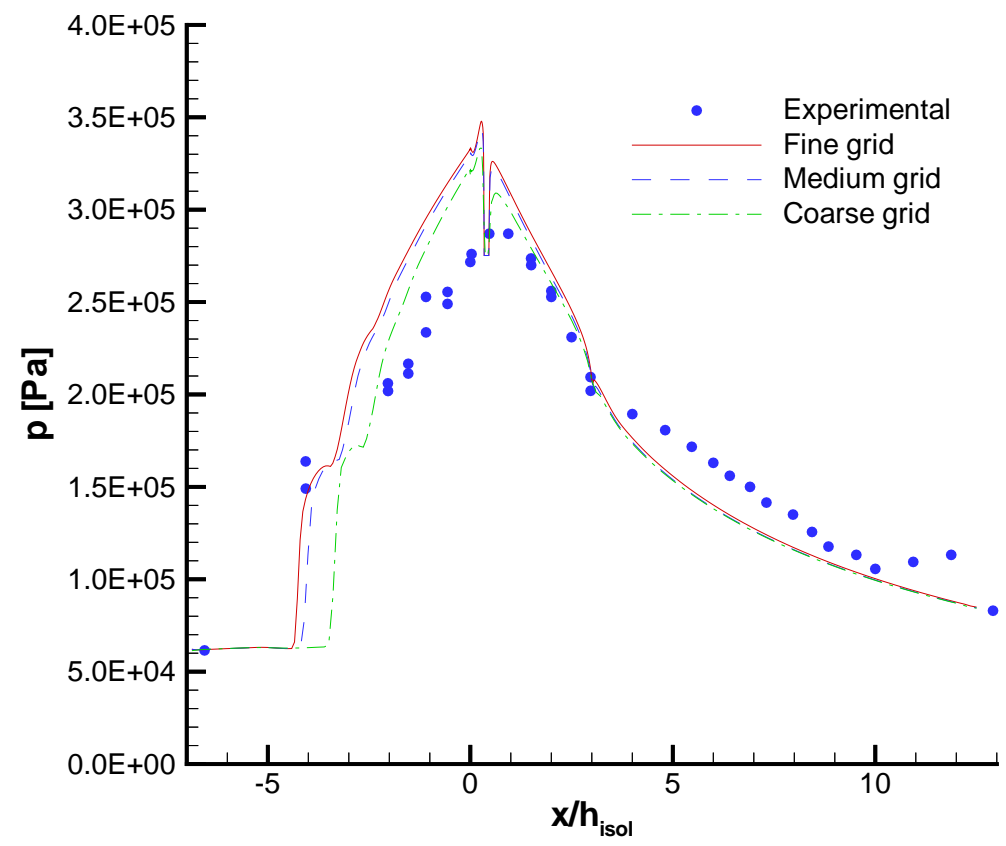

Figure 6: Jet-to-Jet - Effect of grid resolution. 


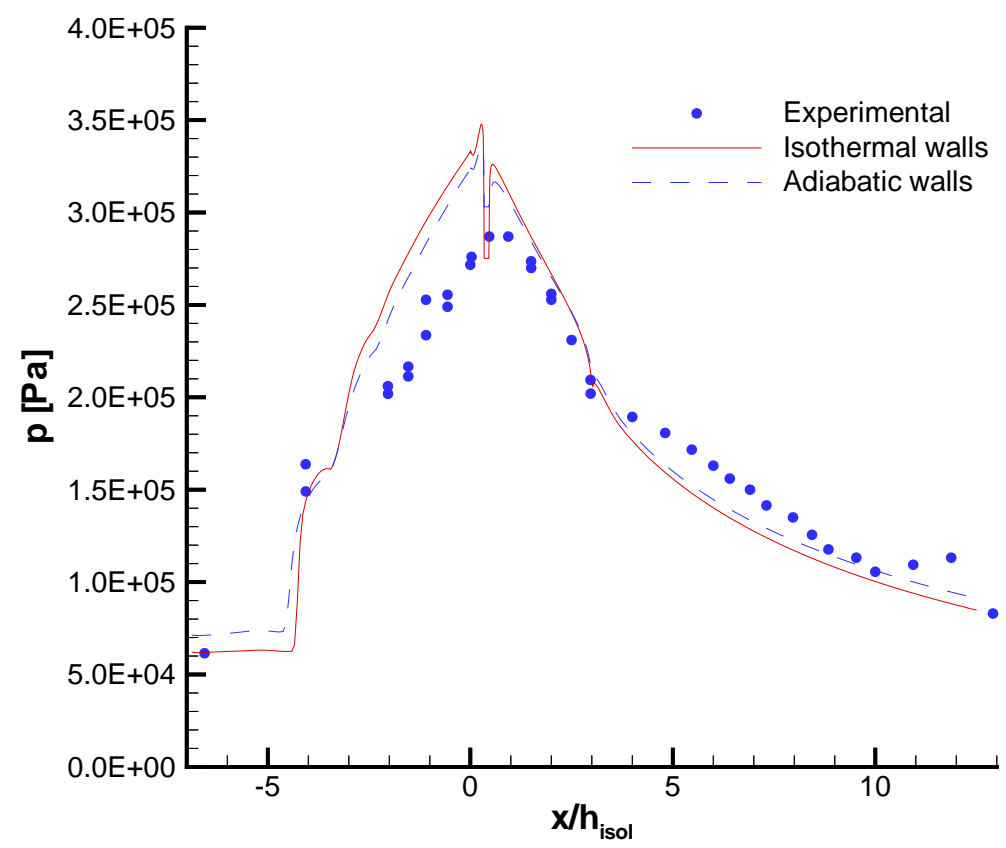

Figure 7: Jet-to-Jet - Sensitivity to wall conditions.

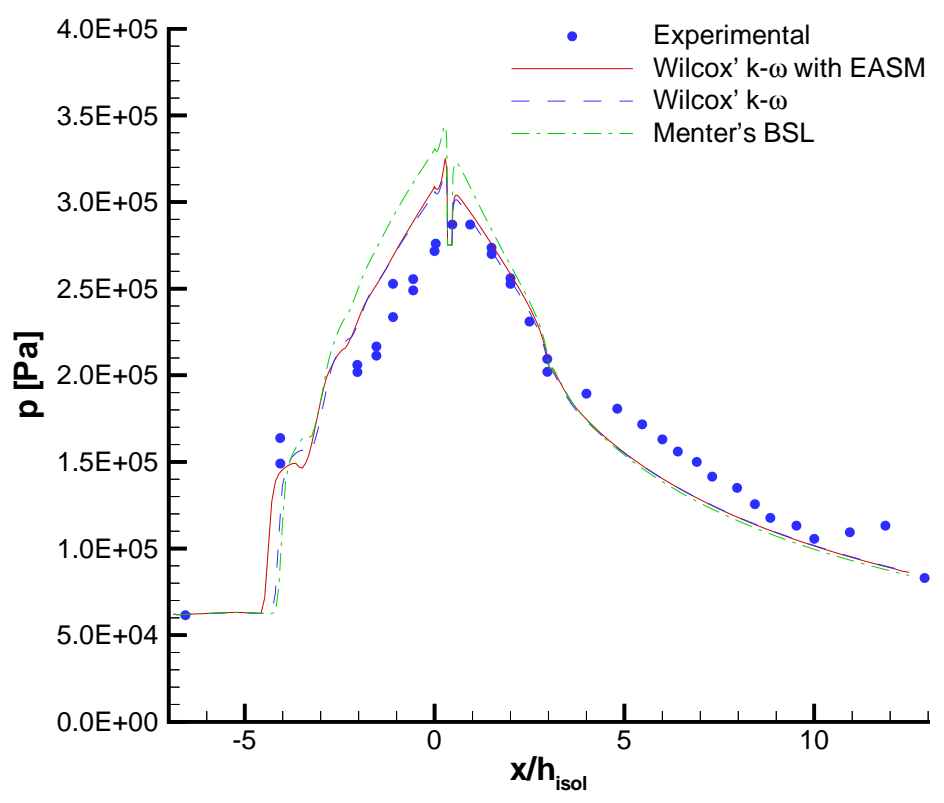

Figure 8: Jet-to-Jet - Effects of turbulence modeling. 

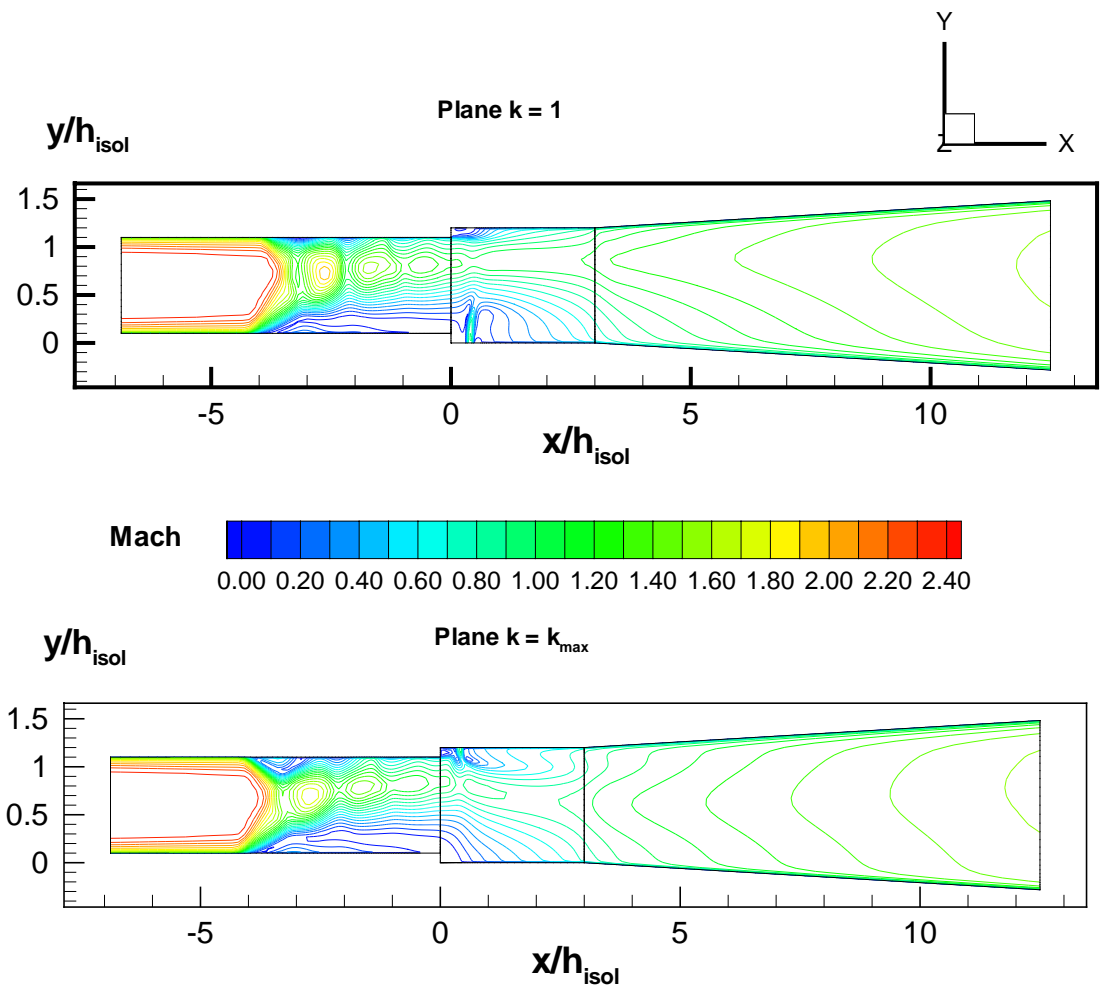

Figure 9: Jet-to-Jet - Mach contours, full height.

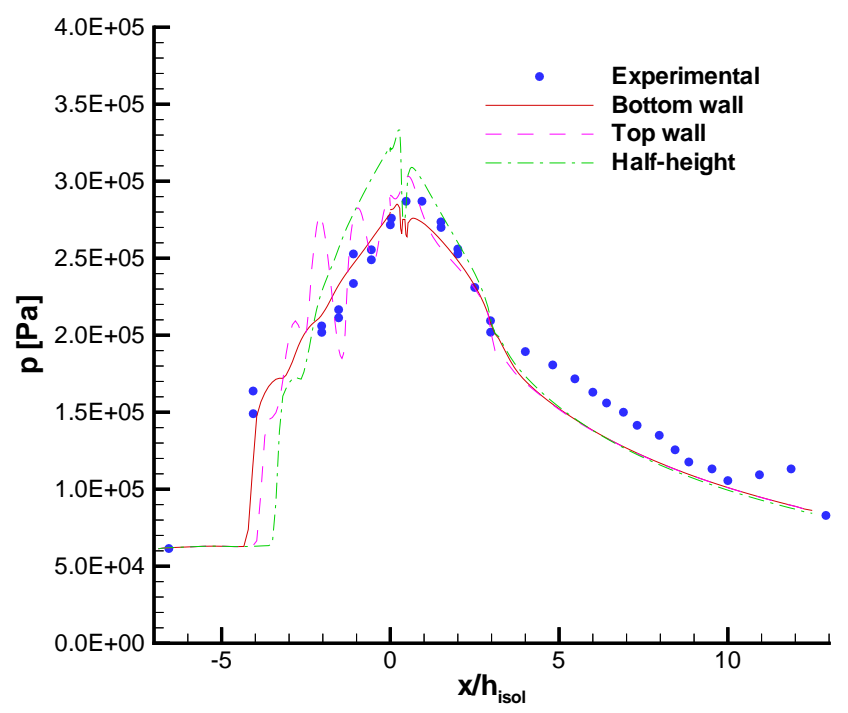

Figure 10: Jet-to-Jet - Wall pressures, full height. 


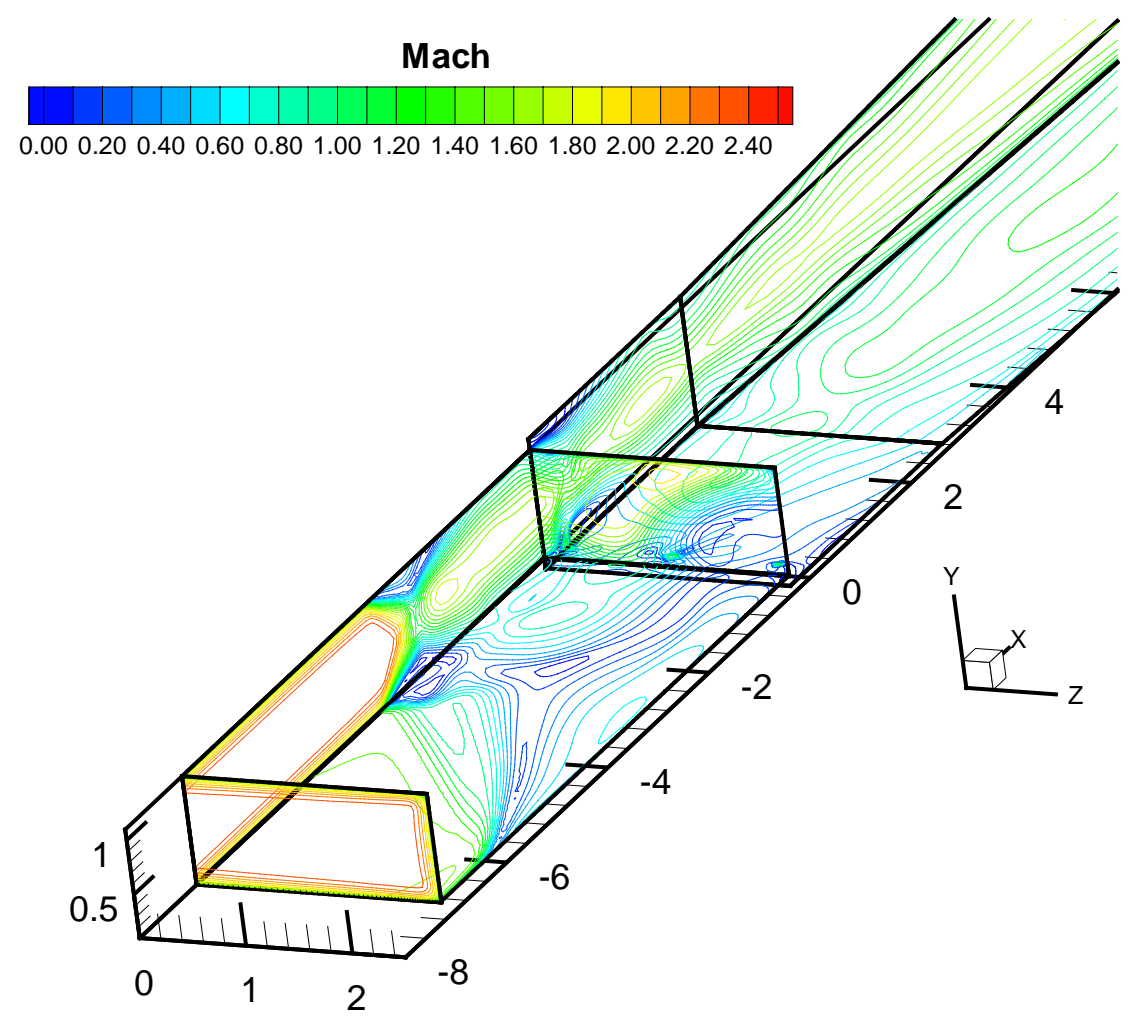

Figure 11: Half-Width - Mach contours.

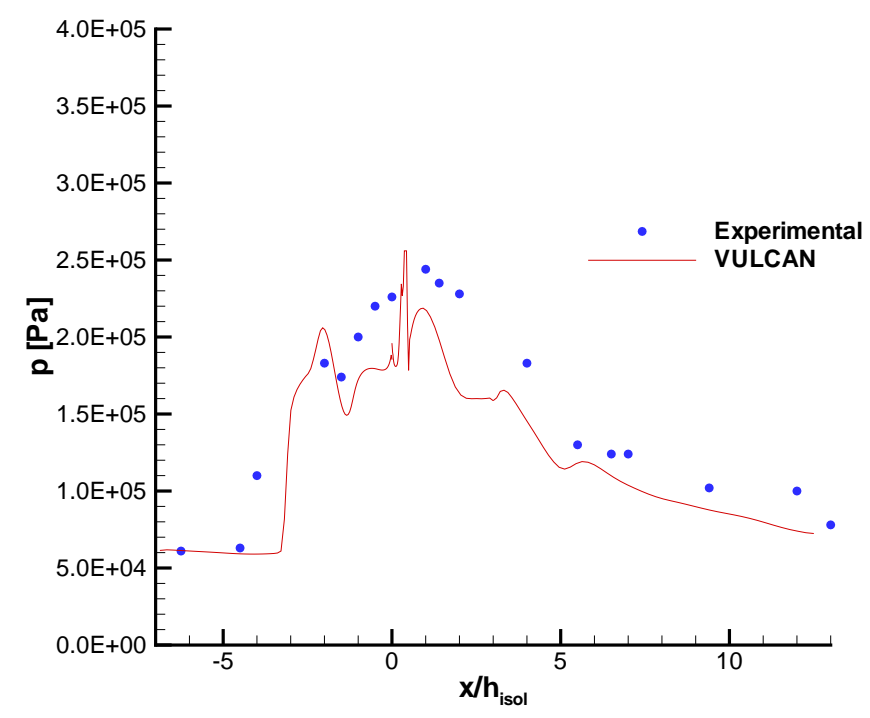

Figure 12: Half-Width - Wall Pressures. 


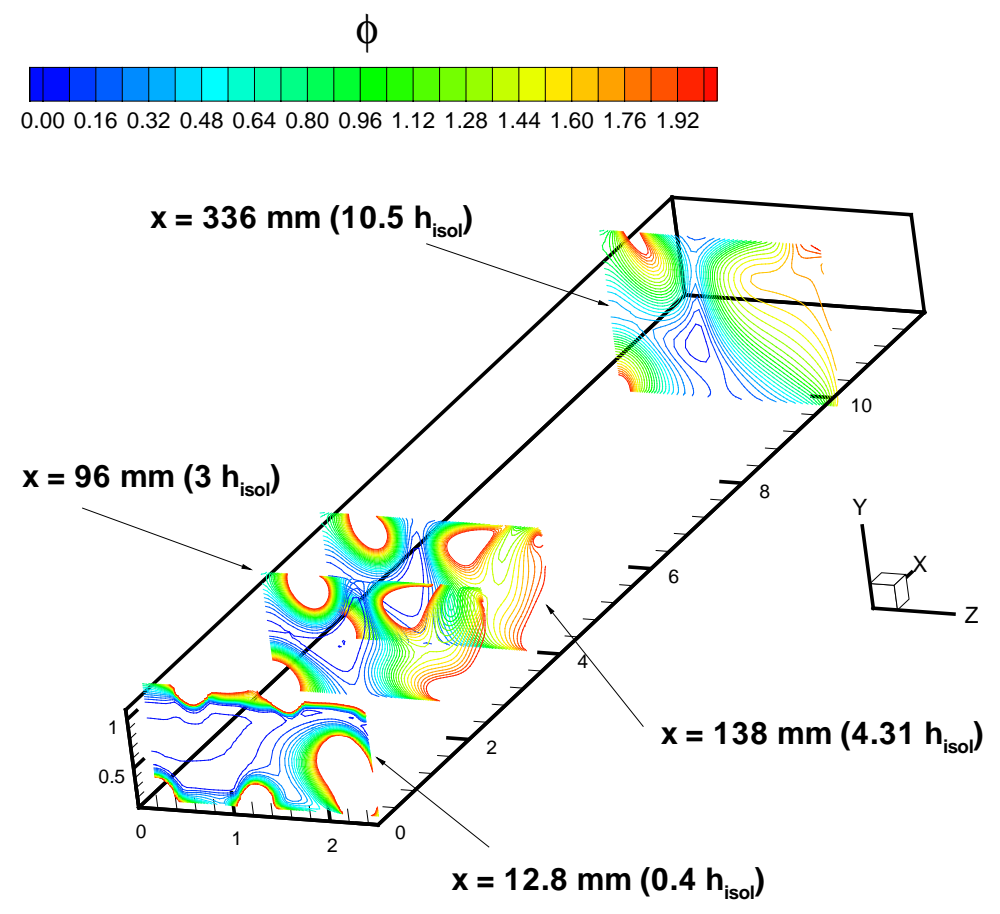

Figure 13: Half-Width - Fuel contours.

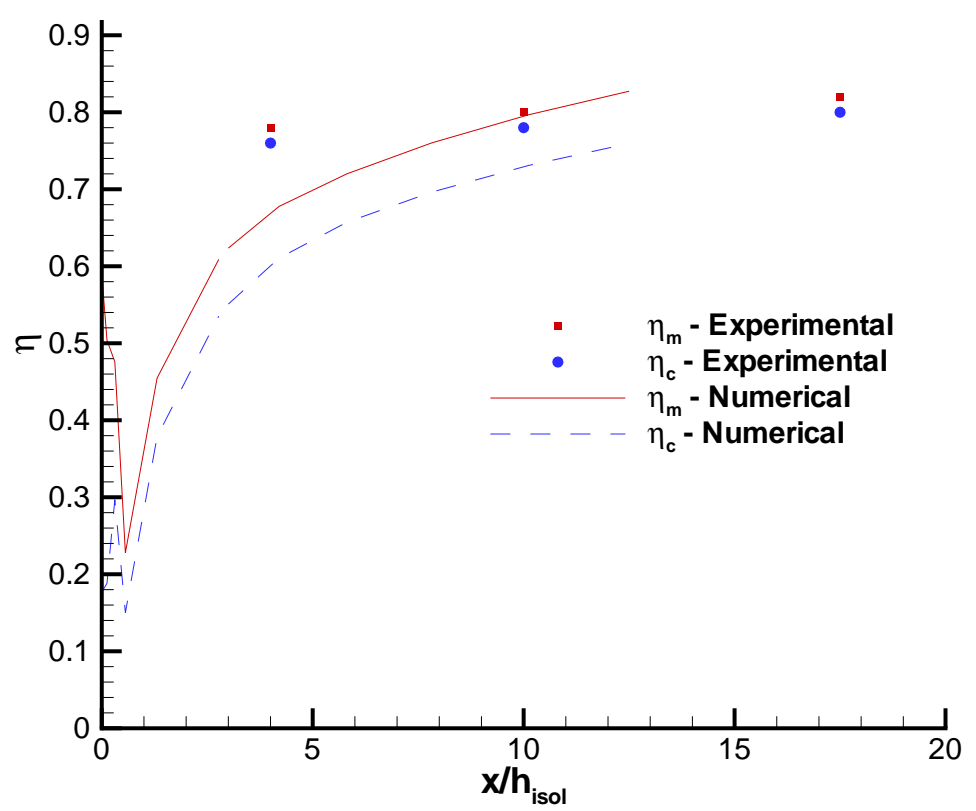

Figure 14: Half-Width - Efficiencies. 